\title{
Hemodynamics of Idealized Carotid Artery
}

\author{
Chaitanya Puritipati ${ }^{1}$, Varun Keri ${ }^{1}$, and Nitesh Kumar ${ }^{1 *}$ \\ ${ }^{1}$ Dept. of Mechanical and Manufacturing Engg., Manipal Institute of Technology, Manipal Academy \\ of Higher Education, Manipal, Karnataka, India
}

\begin{abstract}
The behavior of blood during its flow in human arteries has always been a subject of keen interest. Flow parameters such as pressure, velocity and wall shear stress vary with changes mainly in the geometry of the artery. The work focusses on the variation of wall shear stress along the walls of an Idealized Carotid Artery. Simulations are carried out for both Newtonian and Non-Newtonian behavior of blood. In the Non-Newtonian flow, Carreau-Yasoda model is chosen for the calculations. Steady flow and Transient flow cases are also analyzed where the User Defined Function is interpreted for further calculations. It is observed that recirculation of flow occurs at a critical area in the geometry. The results obtained are compared with the numerical results of Eleyas Shaik. The Wall Shear Stress plots are found to be in close correspondence to those in the thesis.
\end{abstract}

Keywords: Carotid Artery, Wall Shear Stress(WSS), Newtonian Flow, Non-Newtonian Flow, Right Inner Wall (ROW), Right Outer Wall (ROW), Left Inner Wall (LIW), Left Outer Wall (LOW)

\section{Introduction}

The Carotid artery plays a significantly crucial role in the cardiovascular system of the human body. The important function of blood flow to the face, neck and the brain of the human body is performed by the Carotid artery [1]. There are 2 Carotid arteries, one on each side of the neck. Each Carotid Artery bifurcates into 2 divisions, namely the Internal Carotid Artery(ICA) and the External Carotid Artery(ECA). The ICA supplies blood to the brain while the ECA supplies blood to the face and the neck [1]. Hemodynamics of the Carotid artery gives us a better idea about the nature of the blood flowing through the artery and about the challenges faced during the flow in different locations inside the artery. Wall Shear Stress(WSS) is a major factor in analyzing the blood flow characteristics inside the artery [1].

\footnotetext{
*Corresponding author: nitesh.naik@manipal.edu
} 


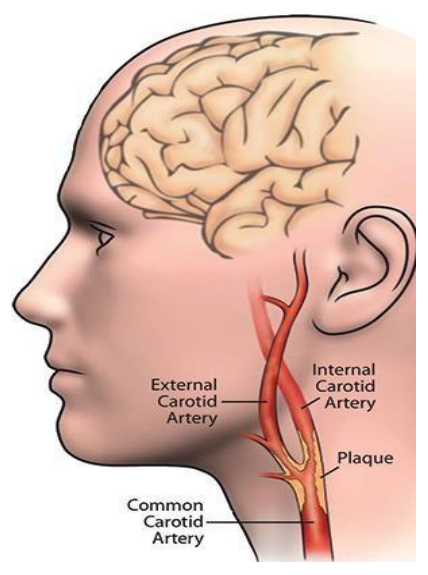

Fig. 1. Carotid Artery Structure

\section{Methodology}

3D idealized model of a carotid artery is modelled using Catia V6, and imported to Ansys CFX 17 to simulate the flow. The model is meshed using inbuilt mesh tool in Ansys workbench and tetrahedral elements are used in this simulation. An element size of $0.39 \mathrm{~mm}$ was selected after grid convergence study. Figure 5 shows the grid convergence of the elements used. Inflation of 4 layers with total thickness of $1 \mathrm{~mm}$ is applied to capture the boundary layer and critical back flow near the wall of the artery. Figure 2 shows the model used in this study. A steady state analysis is performed to compare the results with those in the literature by Eleyas Shaik [1]. The boundary condition applied for steady flow analysis is an inlet velocity of $0.07 \mathrm{~m} / \mathrm{s}$ and an outlet pressure at both outlet surfaces of $0 \mathrm{~Pa}$. Consequently, the boundary conditions for the transient flow analysis is the same outlet pressure of $0 \mathrm{~Pa}$ at both outlet surfaces while the inlet velocity is according to the user defined function. Further, analysis is also done considering different types of flow, i.e., Steady and Pulsatile flow and Newtonian and Non-Newtonian Flow. For the Non-Newtonian type of flow, Carreau-Yasuda model is adopted. The parameters required for it are mentioned in Table 1.

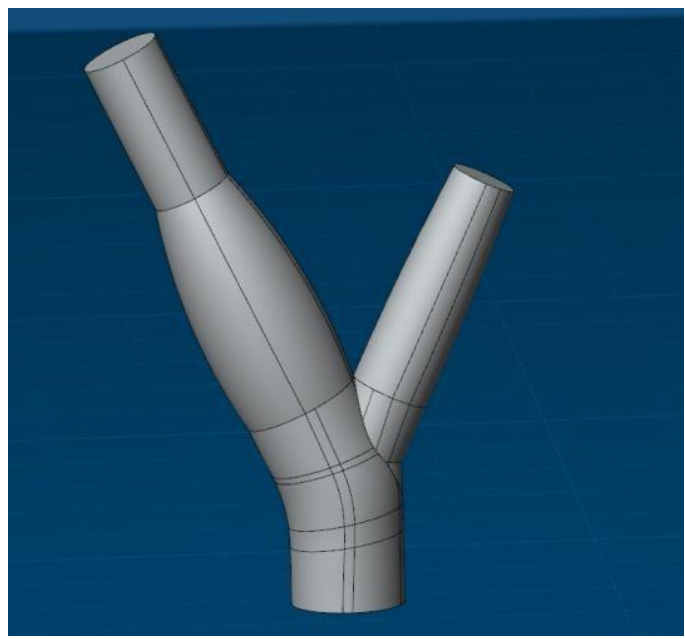

Fig. 2. CATIA model of Idealized Carotid Artery 


\subsection{Software Tools}

The ANSYS Workbench platform upon which a geometric model is created, meshed and appropriate boundaries are identified and marked. With bi-directional CAD connectivity, powerful highly-automated meshing, a project-level update mechanism, pervasive parameter management and integrated optimization tools, the ANSYS Workbench platform delivers unprecedented productivity, enabling Simulation Driven Product Development. Ansys Fluent ${ }^{\circledR}$ is commercially available CFD software widely accepted for its high reliability. The software has different capabilities and functions. Both 2D and 3D models can be tested with laminar, inviscid, or turbulent flows. Steady and unsteady problems can also be handled using this software. Depending on the model and flow type the designer chooses the appropriate flow field type. Fluent also provides mesh flexibility including the ability to solve complex flow problems using unstructured meshes that can be generated about complex geometries with relative ease. Supported mesh types include 2D triangular or quadrilateral, 3D tetrahedral, hexahedral or polyhedral meshes and hybrid meshes. Fluent is flexible to allow us to refine or coarsen a grid based as required. Ansys Fluent ${ }^{\circledR}$ is written in the computer language $\mathrm{C}$ and makes use of the flexibility and variety offered by the language. User defined functions (UDF) written in $\mathrm{C}$ may also be appended to Fluent, thus increasing its versatility. Fluent uses finite volume discretization method in order to convert Partial Differential Equations of conservation of mass and momentum into non-linear algebraic equations [1]. CATIA platform is widely used for modelling various kinds of geometries. The solid part models created in CATIA can be exported to other softwares like ANSYS for further analysis. We have used all the above mentioned softwares for catering to our design and analysis needs.

\subsection{Governing Equations}

Continuous fluid flow is governed by the Navier-Stokes equations. The Navier Stokes equations consist of 3 equations, namely the Continuity equation, the Momentum equation and the Energy equation which is obtained by applying the conservation of the fluid flow. In this study, we assume isothermal conditions for the simulations, hence the energy equation is mentioned here for the sake of completeness. [1]

The principle of mass conservation applied to flowing fluid results in the continuity equation, which can be expressed as

$$
\frac{d \rho}{d t}+\rho \nabla \cdot \vec{u}=0
$$

The momentum equation can be expressed as

$$
\begin{aligned}
& \frac{\partial}{\partial t}(\rho \underset{\sim}{v})+\operatorname{div}(\rho \underset{\sim}{v})=-\nabla p+\operatorname{div}(\tau)+\rho \underset{\sim}{g} \\
& \underset{\sim}{\tau}=\mu\left[\left(\operatorname{grad}(\underline{v})+\operatorname{grad}(\underline{v})^{T}\right)-\frac{2}{3} \operatorname{div}(\underline{v}) \underset{\sim}{I}\right]
\end{aligned}
$$

Where $\rho$ is the density, $\underset{\sim}{v}$ is the velocity, $p$ is the pressure, $\underset{\sim}{\tau}$ is the stress tensor and and $\underset{\sim}{g}$ is the body force. 
In case of pulsatile flow, the boundary condition at inlet is given in the form of the following waveform generated by the User defined function. [2]

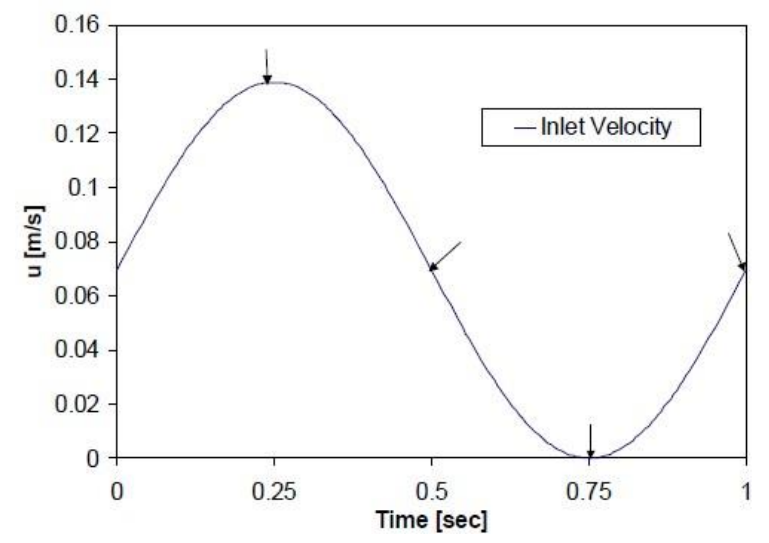

Fig. 3. Inlet Velocity Waveform for Pulsatile flow

\subsection{Non-Newtonian Constitutive Equations}

For the non-Newtonian case, the shear effect on viscosity must be considered. There are various models in several literatures to justify the non-Newtonian behavior of blood, e.g., the Casson model [4]. Yeleswarapu [5] provides a detailed report on these models. In the current investigation, the complex rheological behavior of blood is approximated using the shear-thinning Carreau-Yasuda model, where the apparent viscosity is expressed as a function of the shear rate, as shown in the equation below. [1]

$$
\frac{\mu-\mu_{\infty}}{\mu_{0}-\mu_{\infty}}=\left[1+(\lambda \dot{\gamma})^{a}\right]^{(n-1) / a}
$$

Table. 1 Parameters used for the Carreau-Yasuda Non-Newtonian Model

\begin{tabular}{|c|c|c|c|c|}
\hline $\begin{array}{c}\mu_{\infty} \\
\text { (Pa.s) }\end{array}$ & $\begin{array}{c}\mu_{0} \\
\text { (Pa.s) }\end{array}$ & a & $\mathrm{n}$ & $\begin{array}{c}\lambda \\
(\mathrm{s})\end{array}$ \\
\hline $2.2 \times 10^{-3}$ & $22 \times 10^{-3}$ & 0.644 & 0.392 & 0.110 \\
\hline
\end{tabular}

\subsection{Grid Convergence Test}

Grid convergence test helps us in deciding the optimum value of the design parameters to be chosen for various calculations. A graph is plotted between one of the parameter/result and the number of elements. As the number of elements keep increasing, the value of the parameter becomes almost constant after a certain value. The number of elements after which the parameter becomes constant is considered as the optimum value which is used in the final calculations. The graph for the Grid Convergence test is shown in Figure 5. From the figure, it can be observed that at around 26,000 elements, the inlet pressure reaches a certain value and further increase in the number of elements do not affect the pressure 
value. Hence, the design conditions which gave us 26000 elements will be considered for all further calculations and results.

\section{Results}

\subsection{Three-Dimensional Idealized Carotid Artery:}

The 3-D model of the artery is developed using CATIA software. The model is then exported to ANSYS Workbench for meshing, calculation and further analysis [1].

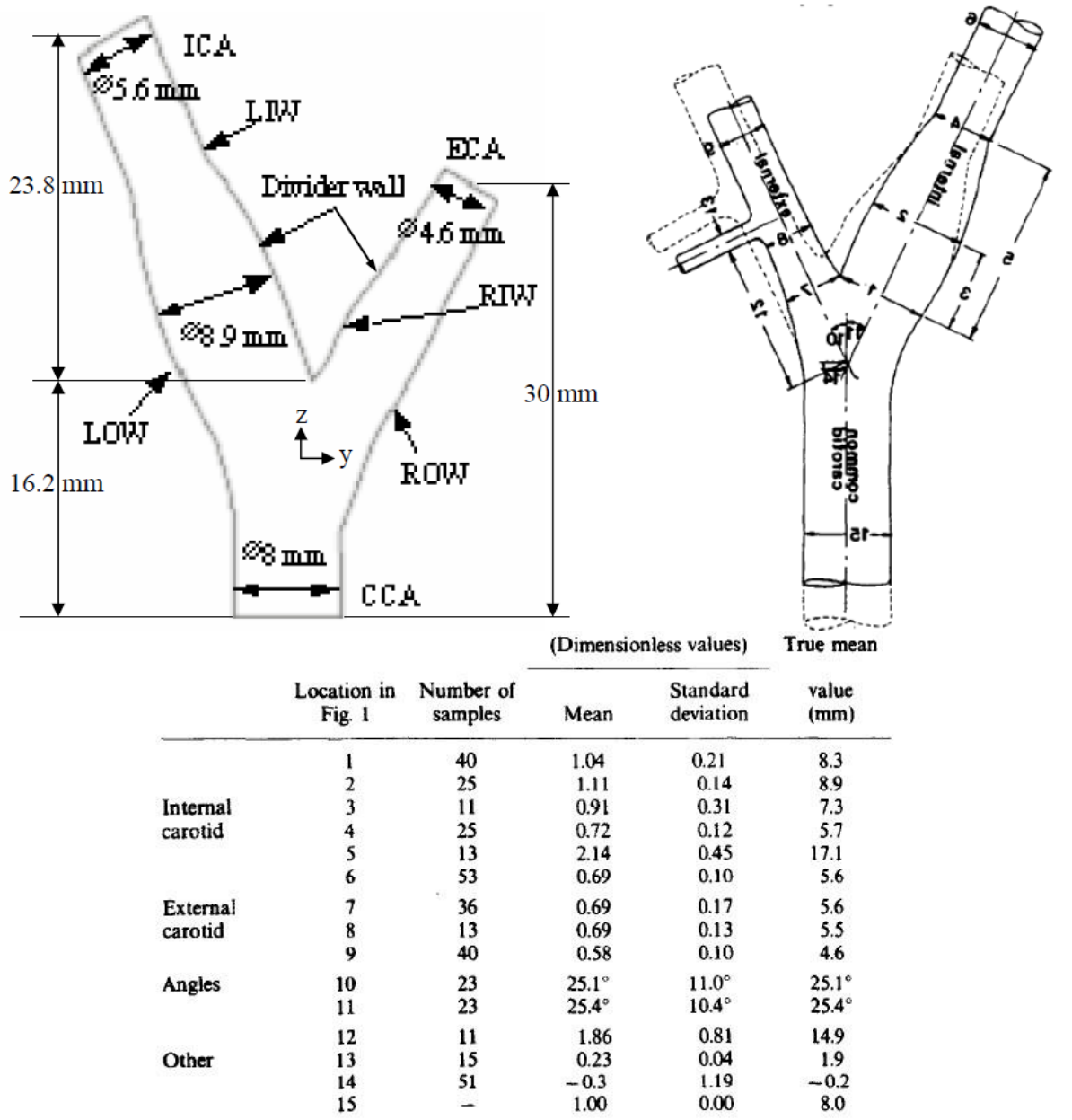

Fig. 4. Schematic Representation of 3D model along with dimensions [2] 


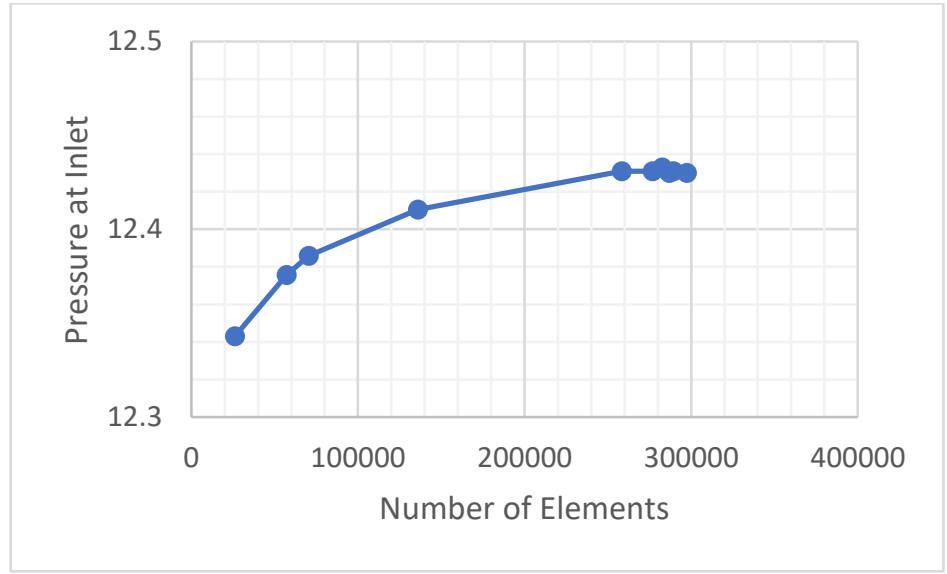

Fig. 5. Grid Convergence Test

\section{Discussion:}

From Fig. 6, we can observe that pressure contour on YZ plane is in close resemblance with that from the literature. Also from the results we can observe that the maximum wall shear stress occurs at the location of the bifurcation. Low wall shear is experienced in the bulging region (Carotid Sinus).

From the Fig. 10, we can observe low velocity in the Carotid Sinus region because of the bulge in the geometry.

Since the inlet surface has multiple contours, average pressure of all surrounding contours were calculated. It was observed that the average pressure at inlet is close to that of the literature value. The velocity vectors emerging out of different planes also were matching with the vectors from different planes in the literature.

Velocity and Pressure graphs in Fig. 11 and Fig. 12 are constructed by taking X axis as $\mathrm{Y}$-distance length by creating lines at different locations such as inlet, ICA entrance, ECA entrance, ICA outlet, ECA outlet.

\subsection{Newtonian Flow Results}
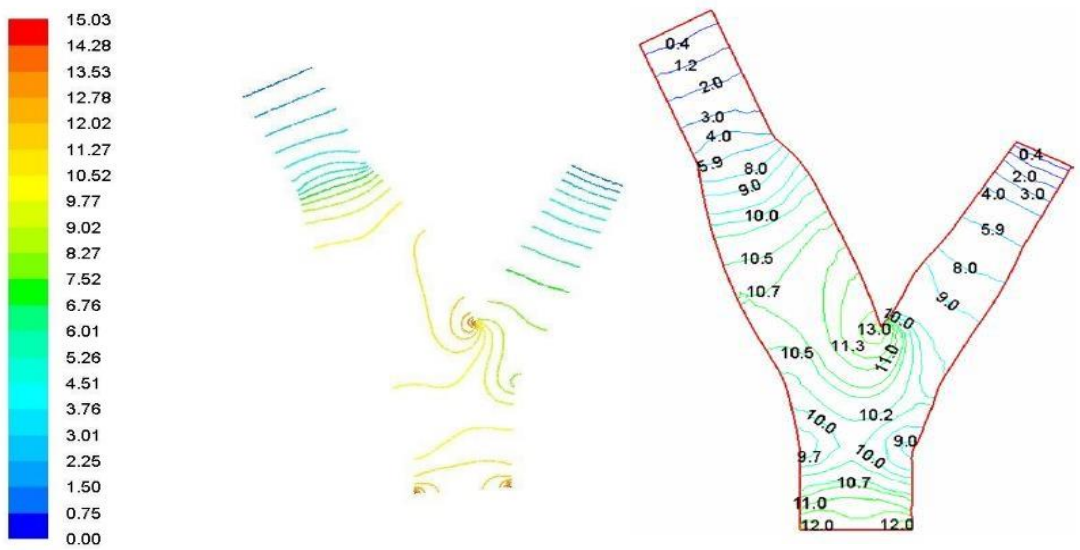

(a)

(b)

Fig. 6. Pressure Contour on YZ plane in (a) Present study, (b) Literature [1] 


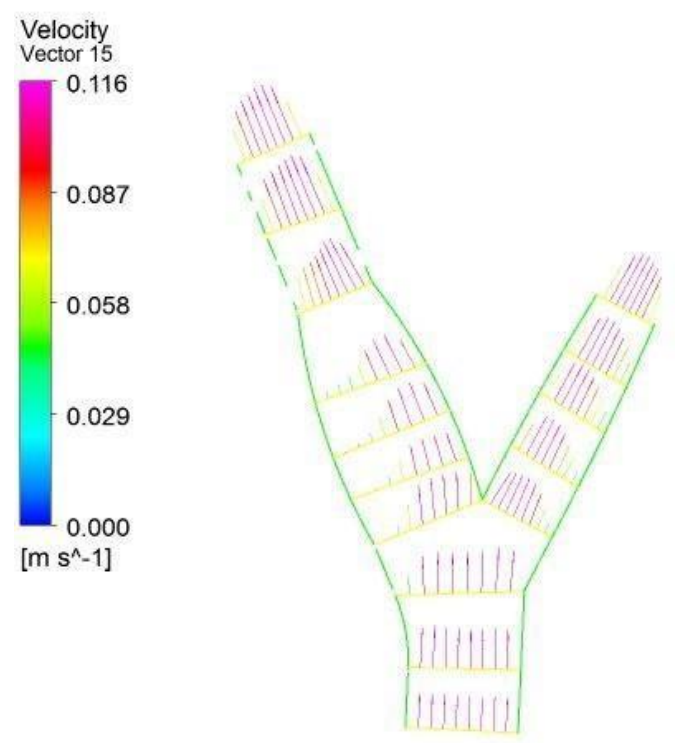

(a)

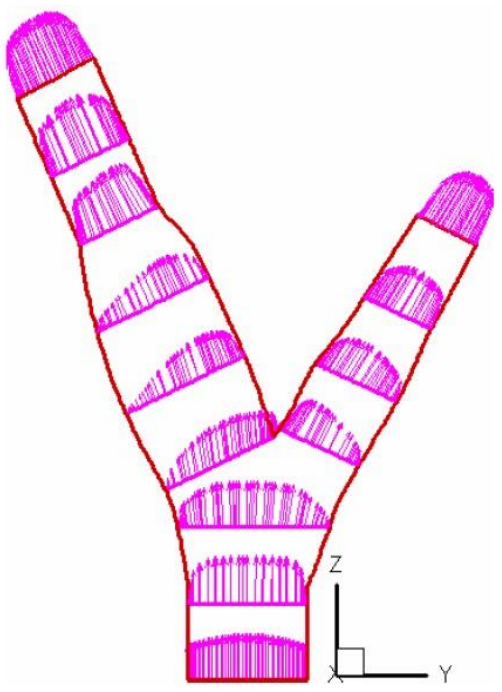

(b)

Fig. 7. Velocity vectors on different XY plane in (a) present study (b) Literature [1]
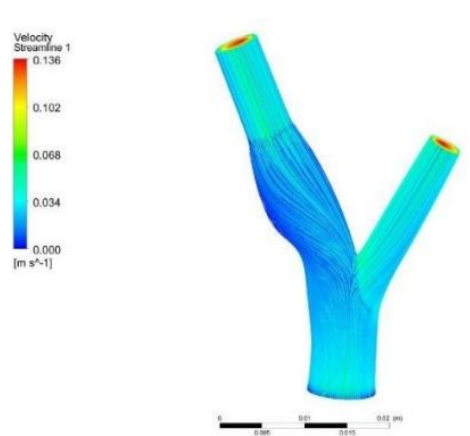

Fig. 8. Streamlines of particles starting from inlet

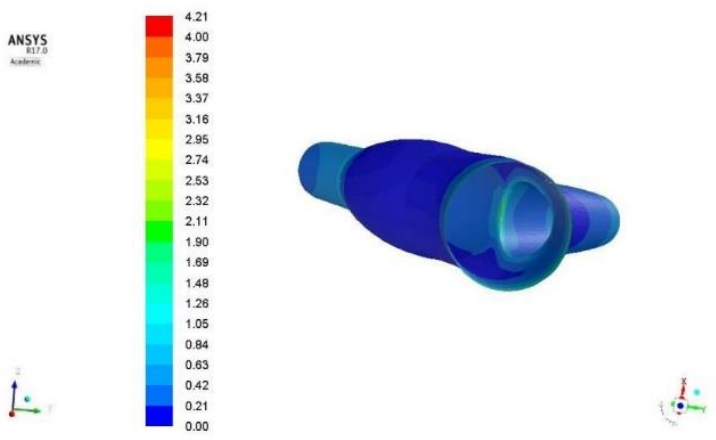

Fig. 9. Wall Shear Contour

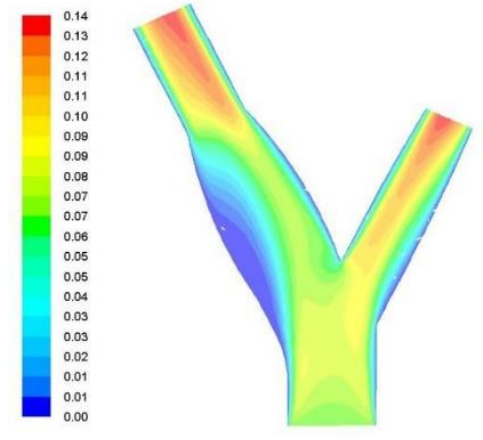

Fig. 10. Velocity contour on YZ plane

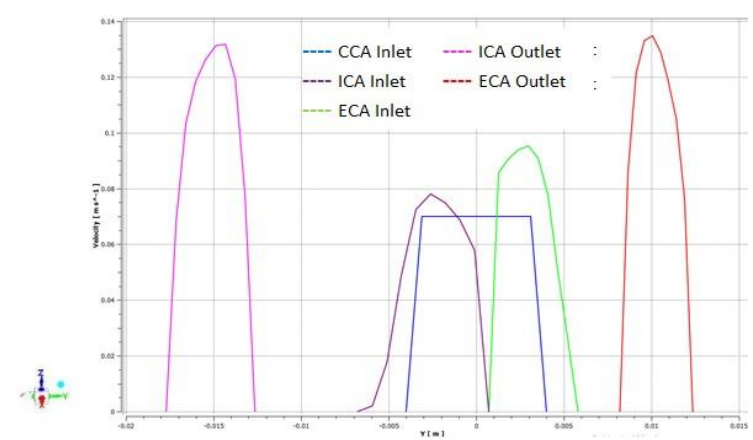

Fig. 11. Velocity vs $Y$ distance at various locations 


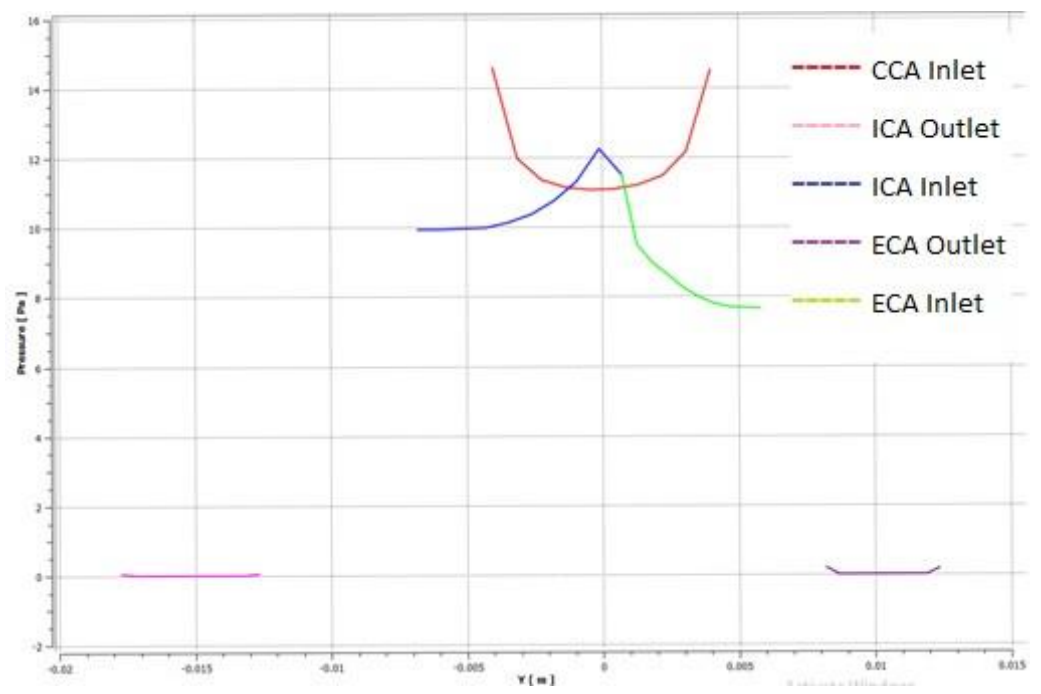

Fig. 12. Pressure vs $Y$ distance at various locations

\subsection{Non-Newtonian Flow Results}

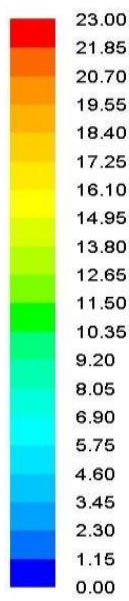

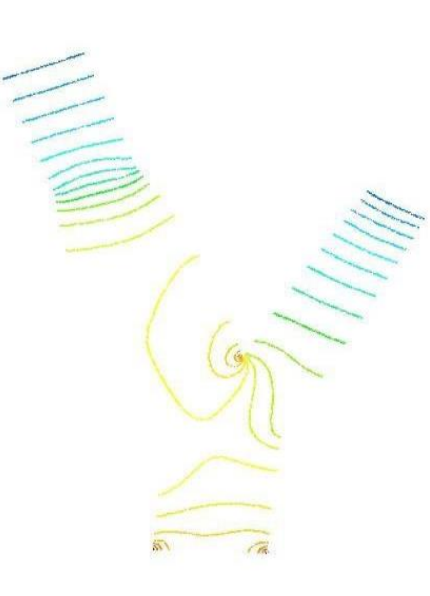

(a)

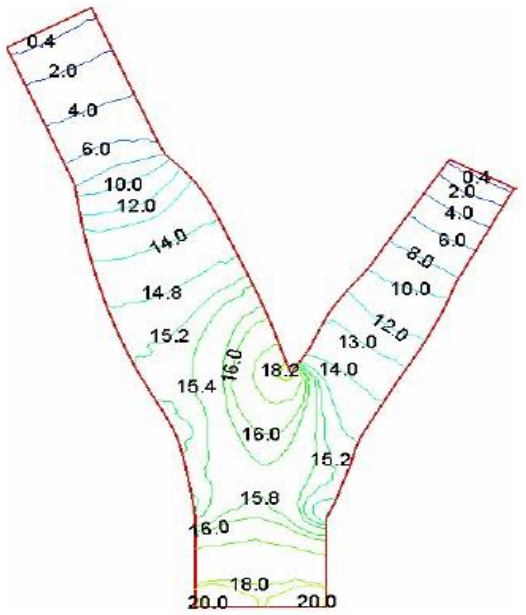

(b)

Fig. 13. Pressure Contour at YZ plane in (a) Present Study, (b) Literature [1] 


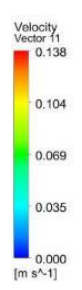

Fig. 14. Velocity contour at YZ plane

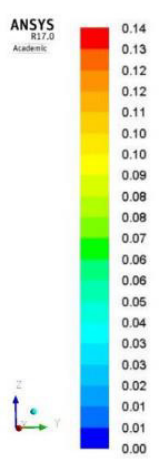

Fig. 15. Wall Shear Contour

\subsection{Pulsatile Flow Results}

In case of pulsatile flow, following changes are made before starting the calculations:

1) The necessary User Defined Function (UDF) for inlet velocity is interpreted

2) The inlet boundary condition accordingly from a constant value to the waveform generated from the UDF.

3) Number of time steps and number of iterations per time step are specified keeping in mind the total cycle time (1 second).

We have opted for 1000 time steps for our calculations.

\section{Discussion:}

A comparative study of the Wall Shear Stress Plots at the boundaries is presented as following:

For Inner Walls, comparison is made with the Wall Shear Stress (WSS) plots from the literature. For Outer Walls, the boundaries are shown to observe the variation of WSS along the respective boundary. From the results, it can be concluded that the WSS Plots obtained are in close correspondence with those from the literature.

As it can be seen from the comparative study in Fig. 17, the WSS is considerably high at the entry. The WSS value at the bulge is very low due to the recirculation of flow. The consequential increase in the WSS value after the bulge can be attributed to the fact that there is a significant decrease in the area which leads to increase in the velocity according to the continuity equation. 


\section{Right Inner Wall}

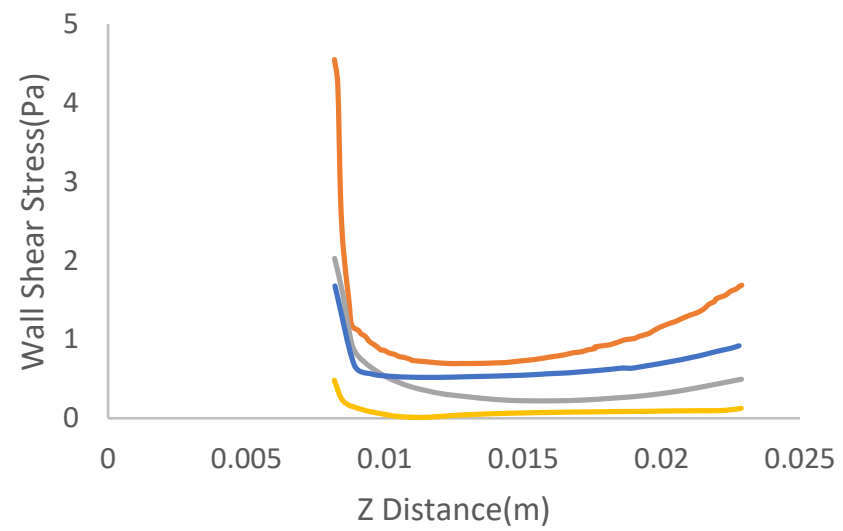




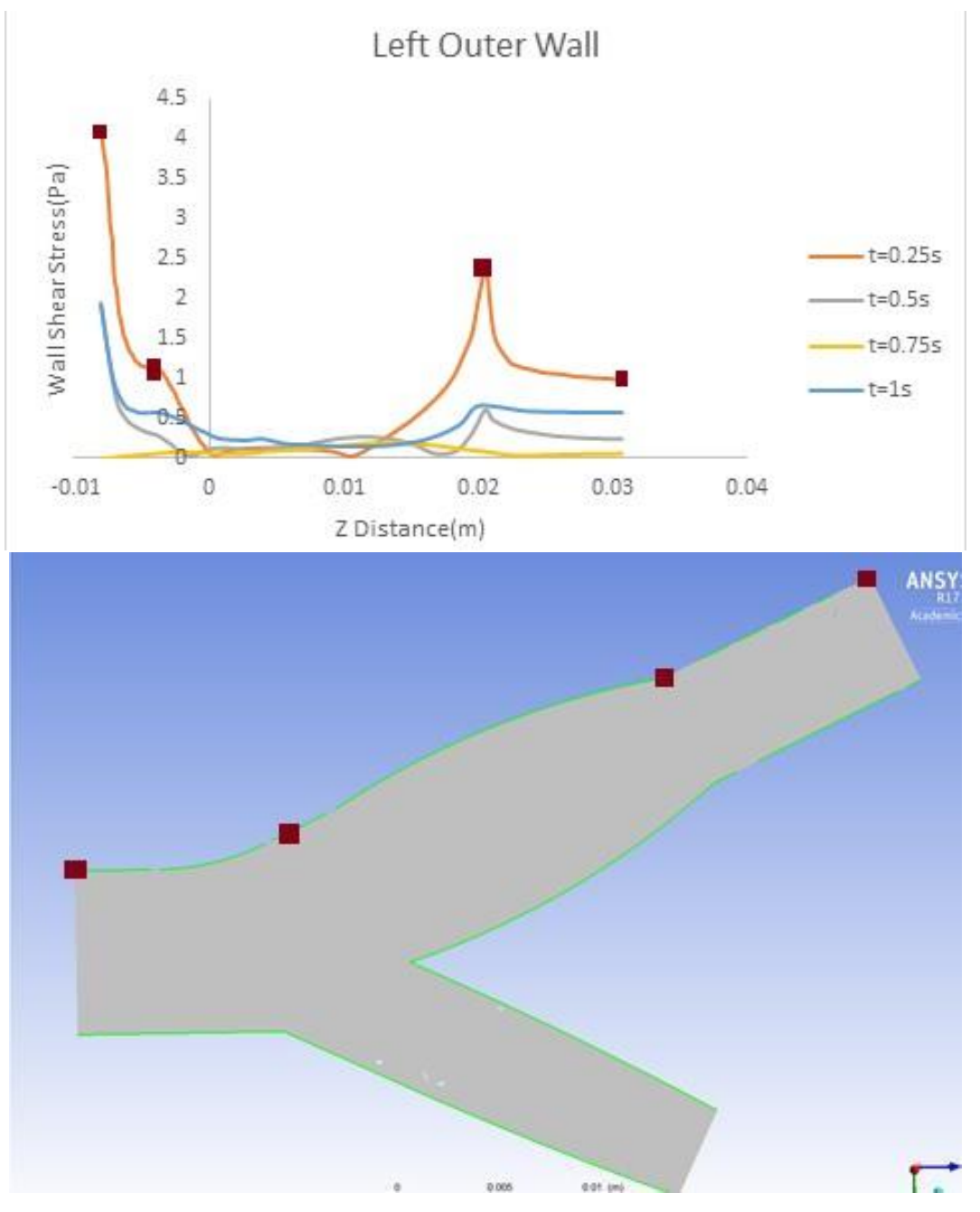

Fig. 17. WSS Plot along LOW 

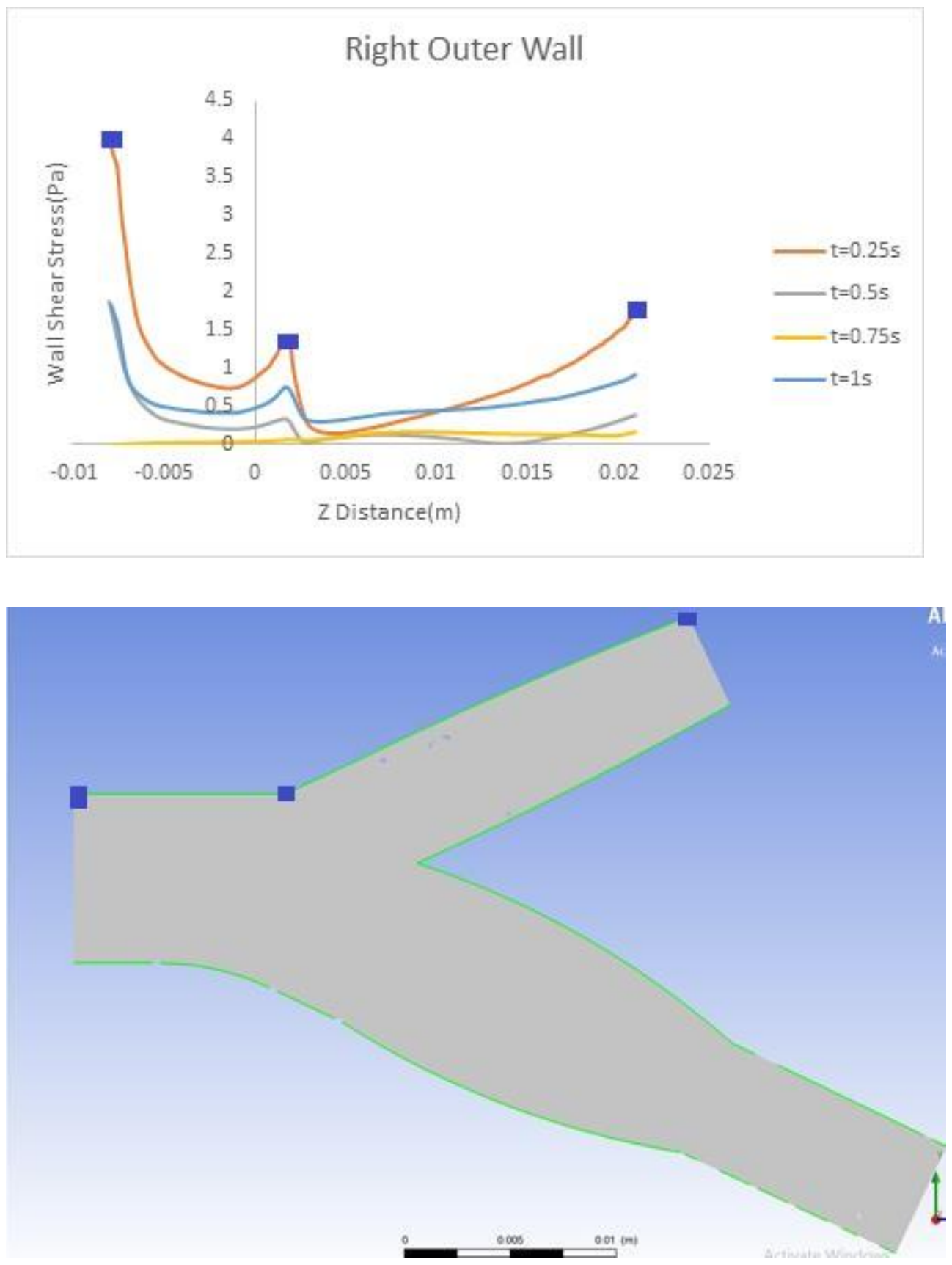

Fig. 18. WSS Plot along ROW 


\section{LEFT INNER WALL}

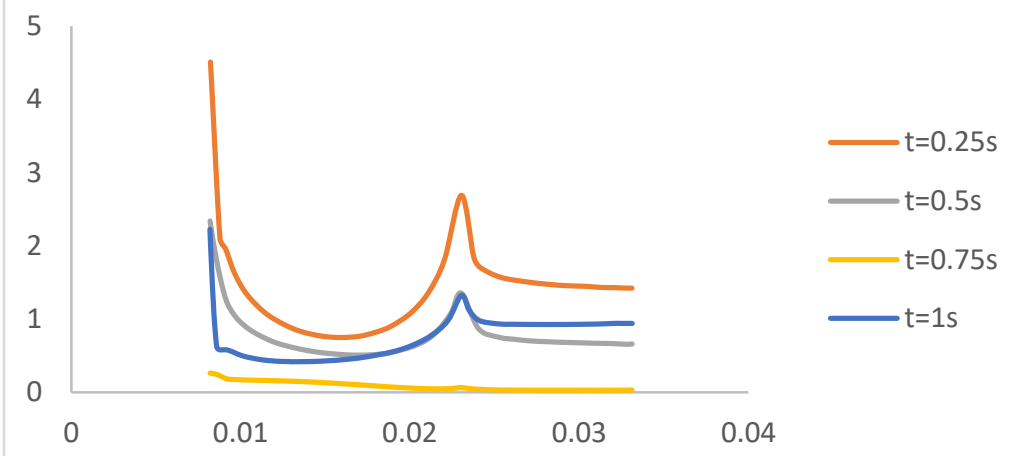

\section{RIGHT INNER WALL}

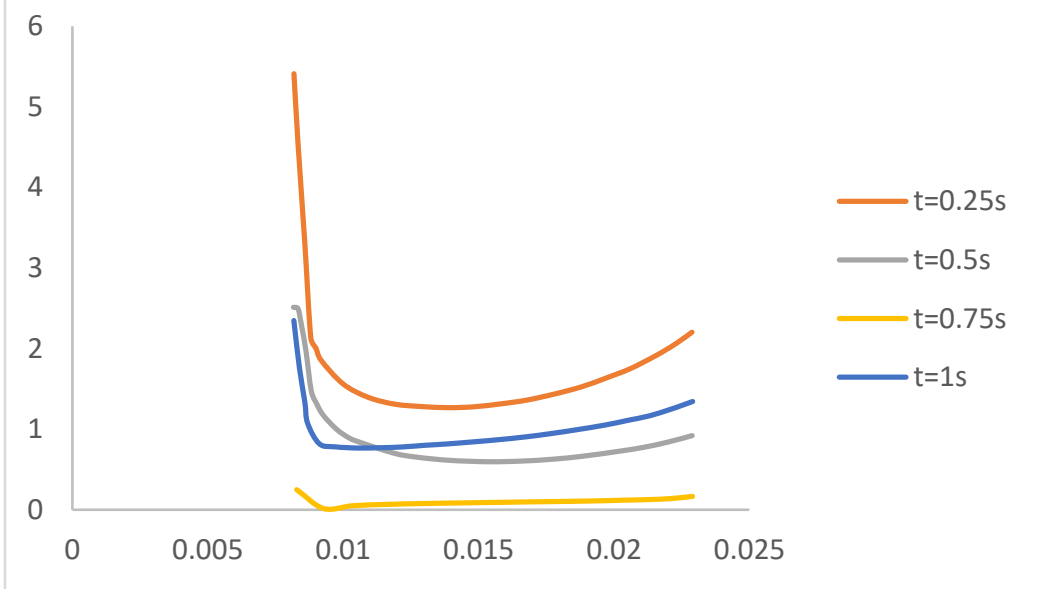

Fig. 19. WSS Plots along Inner Walls

\section{Discussion:}

The above graphs in Fig. 19 depict the behavior of Wall Shear Stress(WSS) with change in Z-distance in meters along both the Left and Right Inner walls. The various values of WSS on the curve are obtained by placing a probe at regular intervals on a line. This line is obtained by the intersection of the X-Y plane located midway through the body along with the body. The various values hence obtained are tabulated for various time steps along with the distances and the above curves are obtained. The nature of curves depends on the geometry of the corresponding wall. In case of the LIW, a steep increase can be observed at the point where the carotid sinus begins. The subsequent reduction in the WSS value can be attributed to the further change in geometry when the bulging portion ends. The following graphs in Fig. 20 depict the behavior of Wall Shear Stress(WSS) with change in Z-distance in meters along both the Left and Right Outer walls. The curves are obtained by following the same process as mentioned in this discussion. The curve of the Right Outer Wall is of considerable importance. The sharpness of the curve at the right outer wall causes the sudden increase in the WSS value due to the stress concentration in the corner. Hence the sudden rise in the WSS value can be observed. 


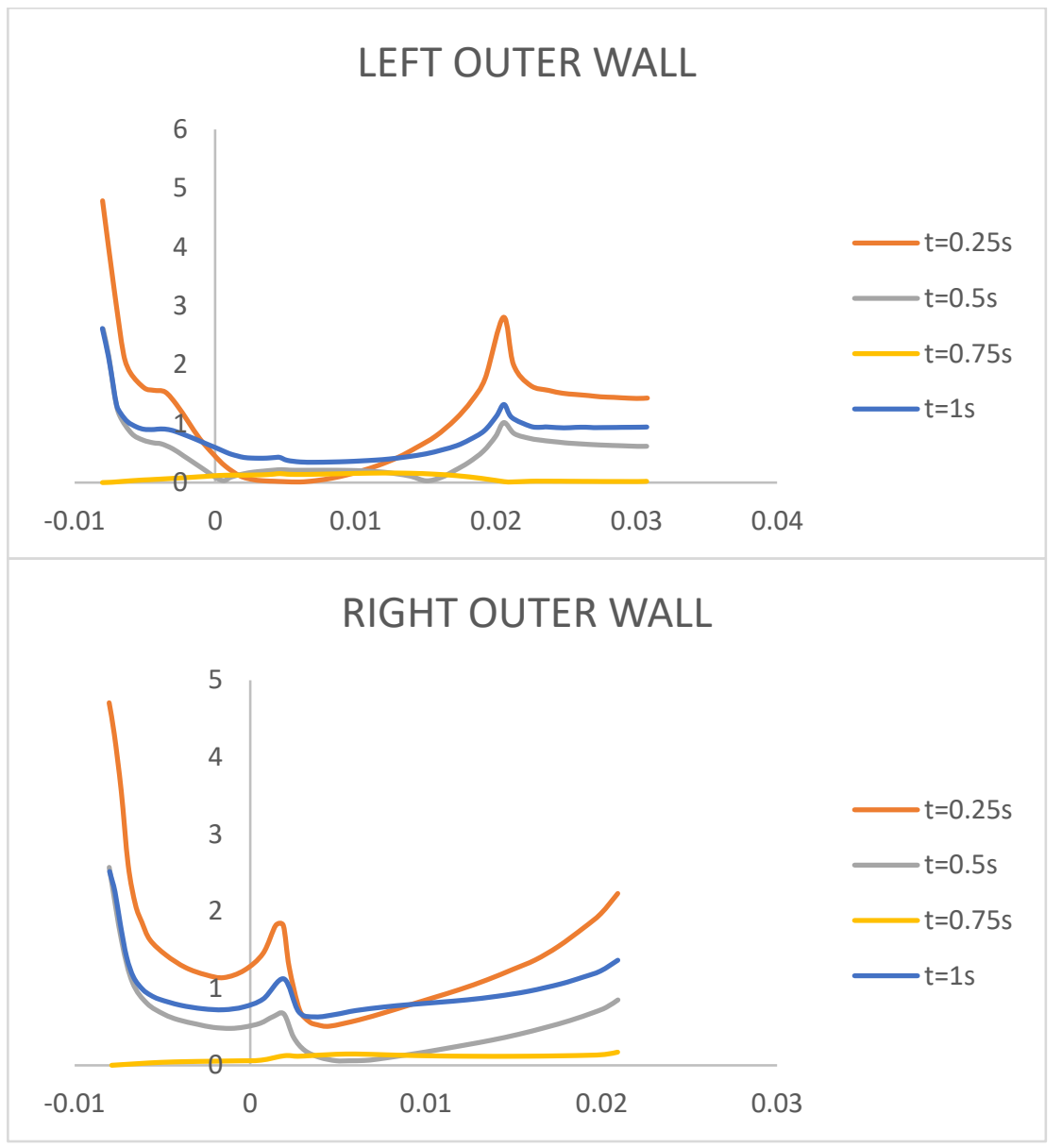

Fig. 20. WSS Plots along Outer Walls

\section{Conclusions}

1. According to the Idealized artery analysis we can conclude that the pressure reduces when there is sudden decrease in area due to bifurcation. This is because the velocity increases with decrease in area according to the continuity equation.

2. Due to the plaque, the heart needs to pump blood with increased pressure to maintain the constant flow rate to the artery.

3. More the constriction in the area of arteries, more will be the pressure at inlet thereby causing more the damage to the artery walls.

4. Flow separation occurs in stenosis because of the adverse pressure gradient.

5. In case of analysis for the Newtonian and Non-Newtonian models, the general trend of variation of the parameters remains almost same with slight deviation in the magnitude.

6. At the carotid sinus region, we observe low velocity and low wall shear stress values.

7. For Newtonian and Non-Newtonian type of flows, there is a change in the maximum wall shear stress value for the same dimensions of the geometry.

8. In case of pulsatile flow analysis, there is considerable variation in the wall shear stress value at different time steps as compared to the steady flow case. 


\subsection{Scope for Future Work}

In our research we have considered the artery to be a rigid structure. But in real scenario, the artery is elastic in nature. Fluid- Structure interaction comes into picture where interface conditions can be specified. Structural deformations change the properties of the blood and also maximum wall shear value will be lesser than that in case of rigid wall. Also we have considered the waveform of the inlet flow to be sinusoidal in nature during the transient analysis. But in actual practice it is pulsatile in nature. The outlet pressure in reality is not zero Pascal because there exists some resistance for the blood flow in the arteries. Actual values of the pressure at the outlet can be specified taking into consideration the blood pressure in different cases. There are different ways to specify outlet pressure such as Resistance, Impedance and constant pressure models. Different user defined functions for different outlet pressure cases can be specified. As a part of further enhancements of analysis, the geometry of the idealized carotid artery can be modified with different percentage of stenosis. The results thus obtained can be compared with real time results from angiograms of patients.

\section{References}

1. "Numerical simulations of blood flow in arteries using fluid-structure interactions," no. July, 2007.

2. "MODELS IN A STENOSIS OF A CAROTID ARTERY," no. December, 2013.

3. R. F. Mabon and D. P. Giddens, "STEADY FLOW IN A MODEL OF THE H U M A N CAROTID BIFURCATION .

4. PART I - - F L O W VISUALIZATION *," vol. 15, no. 5, 1982.

5. Chien, S., Usami, S., and Richard, S., Blood Flow in Small Tubes. Handbook of Physiology, Section 2: The

6. Cardiovascular System, Microcirculation, Part I, Amer. Physio. Soc., Bethesda MD, Vol. IV, 1984, pp. 217-249

7. Yeleswarapu, K. K., "Evaluation of Continuum Models for Characterizing the Constitutive Behaviour of Blood," PhD.

8. Dissertation, Department of Mechanical Engineering, University of Pittsburgh, 1996 